\title{
Comportamiento laboral en época de covid-19.
}

Revisión sistemática

Nayu Mercedes Bolivia-Rebolledo

nboliviar@ucvvirtual.edu.pe

Mirtha Castañeda-Castro

mirtitacc@yahoo.com

Juan Méndez Vergaray

jmvevaluaciones@hotmail.com

Aracelly Angelina Recines -Padilla

aracelly1044@gmail.com

Aurelio Juan Villaverde-Aguilar

juanvillagui@hotmail.com

Edward Flores

eflores5000@gmail.com

Universidad César Vallejo

Lima - Perú

\section{RESUMEN}

En este contexto de emergencia sanitaria a causa de la COVID-19, todos los países enfrentaron cambios educativos, económicos, sociales y sanitarios inesperadas, ocasionando transformaciones en las formas y modalidades de trabajo; generando en las personas un cambio en el comportamiento laboral. Objetivo: El propósito de este estudio es investigar como la pandemia del COVID-19 cambió o influyo en el comportamiento laboral en las personas, en instituciones públicas y privadas. Metodología: este estudio se realizó a través de una revisión sistemática de artículos científicos, entre el 2020 al 2021 relacionados al comportamiento laboral. Esta sistematización de información se ejecutó por medio de una búsqueda intensiva de las principales bases de datos científicos Scopus y Ebsco; además, se utilizó los descriptores de búsqueda o palabras clave: Labor behavior, work behavior, work attitude. Resultados: El proceso de selección según los criterios de exclusión e inclusión de la investigación quedaron 20 artículos para ser analizados a profundidad. Conclusión: El comportamiento laboral durante la epidemia de la COVID19, se ha visto afectada en diferentes ámbitos como la aparición del estrés, la incorrecta posición ergonómica, ansiedad, dificultad en las relaciones matrimoniales, la responsabilidad de adecuarse al trabajo remoto y la atención de su familia.

Palabras clave: comportamiento laboral; conducta laboral; actitud laboral. 


\title{
Work behavior in times of covid-19. Systematic review
}

\begin{abstract}
In this context of health emergency due to COVID-19, all countries faced unexpected educational, economic, social and health changes, causing transformations in the forms and modalities of work; generating in people a change in work behavior. Objective: The purpose of this study is to investigate how the COVID-19 pandemic changed or influenced the work behavior of people, in public and private institutions. Methodology: this study was carried out through a systematic review of scientific articles, between 2020 and 2021 related to work behavior. This information systematization was carried out through an intensive search of the main scientific databases Scopus and Ebsco; In addition, the search descriptors or keywords were used: Labor behavior, work behavior, work attitude. Results: The selection process according to the exclusion and inclusion criteria of the research left 20 articles to be analyzed in depth. Conclusion: Work behavior during the COVID-19 epidemic has been affected in different areas such as the appearance of stress, incorrect ergonomic position, anxiety, difficulty in marital relationships, the responsibility to adapt to remote work and care of his family.
\end{abstract}

Keywords: work behavior; work conduct; work attitude.

Artículo recibido: 02 noviembre. 2021 Aceptado para publicación: 28 noviembre 2021

Correspondencia: eflores5000@gmail.com Conflictos de Interés: Ninguna que declarar 


\section{INTRODUCCIÓN}

El SARS-COV2 es conocido como un síndrome respiratorio, parte del grupo Coronaviridae, conocido con el nombre de COVID-19, se inició casi culminando el año 2019 como un reciente betacoronavirus en seres humanos cercanos a un mercado de mariscos ubicado en Wuhan, China; la aparición de contagio del síndrome respiratorio agudo severo (SRAS) en 2002 a 2003 y el síndrome respiratorio del Medio Oriente (MERS) en 2012, fueron causados por cepas de tipo o variedad de coronavirus que no se ha comprobado la probabilidad de contagio por parte de animales a las personas, como resultado la transmisión de este virus en humanos; en algún momento se opinó que este virus fue transmitido por una especie de animal, la hipótesis del murciélago no ha sido confirmado. Los síntomas de la COVID-19 de acuerdo a la OMS son entre otros: temperatura corporal alta, dolor de articulaciones, problemas estomacales, tos, nauseas; asimismo, se destaca que los pacientes en riesgo de contraer esta enfermedad son aquellas que padecen enfermedades recurrentes de tipo cardiovascular, diabetes mellitus, obesidad; además de los operados de trasplanté de órganos; por otra parte, esta enfermedad puede ser asintomática, leve, moderada o severa (Antezana Llaveta \& Arandia-Guzmán, 2020).

La OMS determinó que se había iniciado una pandemia el 11 de marzo de 2020 e informó que aproximadamente 116 millones habían sido infectados en el mes de mayo de 2021 y 3,4 millones de personas muertas a nivel mundial; este escenario obligó asumir cambios de comportamiento en los trabajadores de la salud; que además, se vieron afectados por el estrés y la ansiedad como consecuencia del impacto a la atención de esta riesgosa enfermedad (Qin et al., 2021 ; Bradley et al., 2021). A nivel mundial, la pandemia de la COVID-19 ha transformado a la sociedad y la vida cotidiana del ser humano, la economía se ha visto afectada en los gobiernos (Mangiavacchi et al., 2021; Krisjane et al., 2020).

La propagación del virus de la COVID-19 generó problemas económicos y sociales a nivel global, obligando a detener el desarrollo de producción comercial como consecuencia se vieron afectados algunas empresas que les obligó a realizar despidos laborales; de esta manera los dueños se adaptaron a subsistir con el servicio de internet para evitar egresos económicos (Yiwen \& Hahn, 2021). Ante esta nueva realidad, el personal de la OMS enuncio brindar un sistema de protocolo en salud en seguridad de los ciudadanos y evitar la transmisión del virus; cancelando la asistencia a lugares públicos 
y privados tales como instituciones, centros comerciales; esto sirvió como una señal para proyectar en direccionar los gastos en productos de primera necesidad, priorizando el bienestar de uno mismo (Tsurugano et al., 2021 ; Bytyçi et al., 2021).

Por otra parte, la gravedad de la pandemia ha convertido el sistema de trabajo de manera drástica en el tiempo, algunos profesionales sin oportunidad de conseguir un bienestar en la parte psicológica a consecuencia del terror a contagiarse, la separación y la cuarentena en casa; además, se ha presentado la intranquilidad por la salud de la familia, la disminución de empleos y ganancias; en la medida que es una amenaza para la humanidad, desarrollando cambios emocionales como el estrés e inquietud de estar distanciado; generando un impacto negativo por la distancia social, en espacio, tiempo y modo de realizar un trabajo; dando lugar a una permanente amenaza de presentar cuadros de ansiedad o inseguridad emocional (Converso et al., 2021); en contraste con los trabajadores externos de salud que adquieren estados de conducta segura para evitar el riesgo de contagio.

Al culminar la primera etapa de paralización en Italia y Europa al inicio del 2020 se elaboraron diversos cambios para contribuir un retorno al puesto de empleo en la etapa 2; el personal que desarrollo actividades desde su casa y empleados de producción que tomaron la decisión de clausurar sus negocios; al respecto, es muy importante adecuar una salud ocupacional para los trabajadores afectados por esta pandemia, brindando la capacitación debida en esta área, en prevención del riesgo de contagio; es por ello, que a partir del 2020, se han realizado estudios sobre aspectos físicos y psicológicos, como resultado de la exposición al COVID-19 (Bogliacino et al., 2021). Además, ante la apreciación de amenaza de contagio, se ha previsto un comportamiento seguro referente al campo laboral de la salud en la pandemia de COVID-19; es por ello, que a partir de febrero de 2020, los expertos han considerado el título de seguridad advertida, el temor a ser contaminado y el uso de indumentaria como protección para el personal de salud; asimismo, la seguridad organizacional se interpreta en el seguimiento del riesgo biológico, está aportación no establece la satisfacción de entender las percepciones de seguridad en los empleados, la condición de seguridad debe estar relacionada, la garantía de la estrategia de medidas de seguridad asumida a la circunstancia de seguridad psicológica (Converso et al., 2021; Bogliacino et al., 2021). 
Con anterioridad a la pandemia de COVID-19 existía una carencia de personal sanitario con documentos actualizados, la OMS calculó una escasez de 18 millones de empleados de salud para 2030 en atender los objetivos de desarrollo sostenible y metas de alcance en el sector salud determinadas por la OMS; al respecto, se han emitido informes de política que indica la dirección, con su respectivo plan de acción europeo para fortalecer las habilidades y servicios de salud pública; además, se ha investigado la cobertura sanitaria universal y estrategia acerca de los recursos humanos sanitario, ante la escasez de trabajadores a consecuencia del desplazamiento de los profesionales sanitarios, cesantes o abandono de puesto de trabajo de salud pública y el desconocimiento de información acerca en desempeñar la función en salud pública. Se ha convertido en una urgencia por la Pandemia de COVID-19; para enfrentar esta problemática la OMS propone recopilar información sobre la fortaleza laboral transformando en un bien público mundial con la finalidad de prevenir la enfermedad y la recuperación de la pandemia de COVID-19 (Krasna et al., 2021).

Sin embargo, algunos empleados toman la decisión de acudir a trabajar en un estado de salud deplorable y enfermos, generando resultados adversos contra la salud en ellos, por causas de tiempo disminuido para su total recuperación y evitar el contagio de infecciones de sumo cuidado donde se ha observado la propagación de COVID-19; aunque en algunos lugares de Europa, se estima que un 40\% laboran una vez al año (Reuter et al., 2021). Asimismo, en América latina, el ámbito laboral en tiempo de pandemia del coronavirus 2019 ha influido en la rutina de trabajo, como nueva modalidad de realizar las actividades desde su hogar llamado el trabajo a distancia o el teletrabajo desde a mediados de marzo de 2020, ejecutando un proceso de transformación de lugar o ambiente, en cumplimiento de lineamientos y responsabilidades; en este contexto, debido a que se cuentan con menos recursos sanitarios aplicaron restricciones laborales en empresas públicas y privadas; al respecto, el informe de la Comisión Económica para América Latina y el Caribe indica que una quinta parte de empleados han trabajado desde su casa en la pandemia (SandovalReyes et al., 2021).

El propósito de este estudio es analizar como la pandemia de la COVID-19 cambió el comportamiento laboral en las personas, teniendo la importancia de asumir nuevos retos con el plan de protocolo en prevención al contagio de este virus que representa nocivo hacia la salud en el ámbito social, económico y político. El ser humano busca ser 
productivo en satisfacción a los consumidores o clientes, marcando el equilibrio en subsistir en beneficio de una economía adecuada.

\section{ESTRATEGIAS METODOLÓGICAS O MATERIALES Y MÉTODOS}

En el presente trabajo se realizó una revisión sistemática de artículos académicos del periodo 2019 al 2021 relacionado con el comportamiento laboral y COVID-19. Esta recopilación de información se realizó a través de una búsqueda exhaustiva en las principales bases de datos científicos: Scopus, Ebsco, se tuvo por conveniente utilizar los descriptores de búsqueda o palabras clave: Labor behavior AND pandemic, Work behavior AND COVID-19, Work attitude AND social distancing.

Esta búsqueda se realizó principalmente teniendo en cuenta artículos de revistas indexadas, donde proporcionaron información acerca del tema, se logró establecer criterios que pudieran tener abordajes cuantitativos y cualitativos.

Criterios de inclusión: Para efectuar la pesquisa se tuvo en atención los artículos concernientes al Comportamiento laboral en época de COVID-19. Asimismo, en la investigación se aplicó únicamente en artículos científicos de revistas indexadas en diversos niveles establecidos en la categoría de publicación proveniente en artículos en el idioma inglés.

Criterios de exclusión: se logró identificar 134 artículos de acceso abierto relacionados al tema, en el primer filtro fueron discriminados por duplicidad de información 34 artículos obteniendo 100 artículos científicos. Tras realizar el proceso de selección según los artículos excluidos a nivel de título, resúmenes y palabras claves, quedaron 60 artículos y los artículos completos excluidos por incumplir los criterios de inclusión declarados en la metodología fueron 39, quedando 20 artículos incluidos para el estudio de comportamiento laboral, como se aprecia en la figura 1. El proceso de codificación de datos se realizó transfiriendo la información a una matriz, respecto al nombre del artículo, año de publicación, categorías, enfoques, tipo de investigación, objeto de estudio, población, muestra investigada e instrumento aplicado en el estudio; transfiriendo la información a una matriz.

La táctica para investigar se fundamentó en la recolección de investigación que revelaban al objeto de estudio; comportamiento laboral que fueron examinados mediante el texto completo y acceso abierto, para ser especificados y sistemáticamente explorados en tablas de datos y referenciado en el gestor Mendeley. 
Figura 1. Adaptación de PRISMA, diagrama de flujo (Moher et al., 2009).

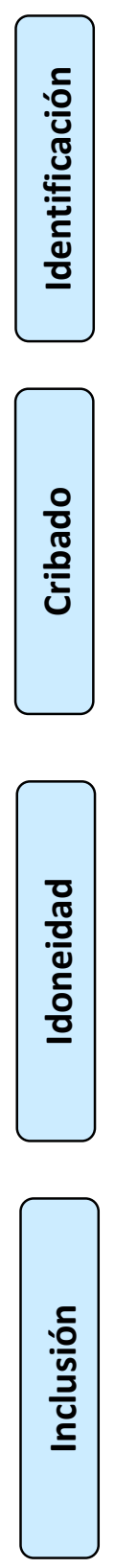

Registros identificados mediante la búsqueda en Scopus, Ebsco $(\mathrm{n}=104)$
Registros adicionales identificados en otras bases de datos $(n=30)$

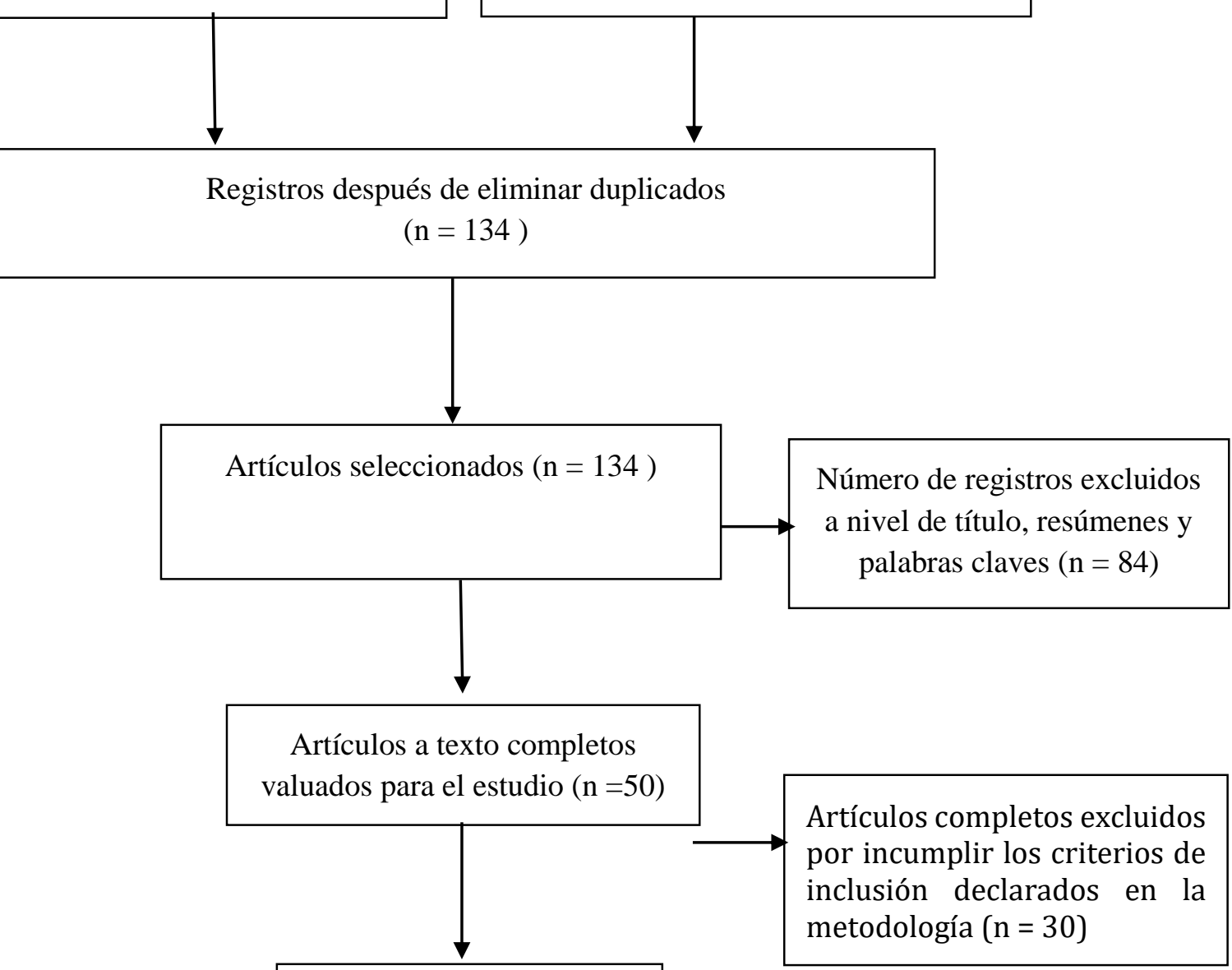

Artículos incluidos en el estudio $(n=20)$ 


\section{RESULTADOS Y DISCUSIÓN}

Tabla 1. Publicaciones que cumplen con los criterios de inclusión

\begin{tabular}{|c|c|c|c|c|c|c|c|}
\hline \multirow[b]{2}{*}{ No. } & \multirow[b]{2}{*}{ Autor/es } & \multicolumn{3}{|c|}{$\begin{array}{c}\begin{array}{c}\text { Comportamiento } \\
\text { laboral }\end{array} \\
\end{array}$} & \multirow[b]{2}{*}{$\begin{array}{l}\text { Tipo de estudio, } \\
\text { población y muestra }\end{array}$} & \multirow[b]{2}{*}{$\begin{array}{l}\text { Instrumento, } \\
\text { técnica o } \\
\text { metodología } \\
\text { empleada }\end{array}$} & \multirow[b]{2}{*}{ Aporte } \\
\hline & & 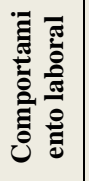 & 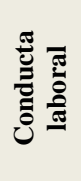 & 恶要 & & & \\
\hline 1 & (Awano et al., 2020) & & $\mathrm{X}$ & & $\begin{array}{l}\text { Cuantitativo, } \\
1964 \text { trabajadores. }\end{array}$ & Cuestionario. & $\begin{array}{l}\text { En la presente investigación se realizó } \\
\text { un estudio el nivel de problemas de } \\
\text { ansiedad, depresión y el estado } \\
\text { emocional, a causa de la pandemia de } \\
\text { COVID-19. El personal manifestó un } \\
\text { grado alto de problemas psiquiátricos, se } \\
\text { debe dar tratamiento psicológico en } \\
\text { beneficio a los trabajadores. }\end{array}$ \\
\hline 2 & $\begin{array}{l}\text { (Hosokawa \& } \\
\text { Katsura, 2021) }\end{array}$ & $X$ & & & $\begin{array}{l}\text { Cuantitativo, } \\
1414 \\
\text { estudiantes de } \\
\text { primaria. }\end{array}$ & Cuestionario & $\begin{array}{l}\text { Según el aporte del presente trabajo, se } \\
\text { ubicó la influencia de los padres de } \\
\text { familia en el contexto de las actividades } \\
\text { laborales, la vivencia en los cambios de } \\
\text { conducta de los niños. Se debe realizar } \\
\text { programas sociales en dar un } \\
\text { seguimiento psicológico en el control de } \\
\text { emociones para mantener el desarrollo } \\
\text { humano de la niñez en sus etapas. }\end{array}$ \\
\hline 3 & $\begin{array}{l}\text { (Nemteanu \& } \\
\text { Dabija, 2021) }\end{array}$ & $X$ & & & $\begin{array}{l}\text { Cuantitativo, } 850 \\
\text { empleados. }\end{array}$ & Cuestionario. & $\begin{array}{l}\text { El marketing es la que se encarga de } \\
\text { llevar un proceso de desarrollar un buen } \\
\text { comportamiento organizacional } \\
\text { incentivando a los trabajadores, en } \\
\text { realizar actividades de producción; por } \\
\text { consiguiente, se ha visto afectada en la } \\
\text { parte emocional debido a la emergencia } \\
\text { mundial. El efecto de la disminución en } \\
\text { la persona causa el desgano de seguir } \\
\text { llevando una rutina satisfactoria, es muy } \\
\text { importantes obtener nuevas estrategias y } \\
\text { alcances de mejora en beneficio a la } \\
\text { empresa y sus trabajadores. }\end{array}$ \\
\hline 4 & $\begin{array}{l}\text { (Magnavita et al., } \\
\text { 2021) }\end{array}$ & $X$ & & & $\begin{array}{l}\text { Cuantitativo, } 905 \\
\text { trabajadores }\end{array}$ & Cuestionario & $\begin{array}{l}\text { El trabajo a distancia se ha desarrollado } \\
\text { desde hace mucho tiempo en diferentes } \\
\text { sectores, con la llegada de la pandemia } \\
\text { se formalizo esta modalidad de laborar } \\
\text { desde su casa, respetando el aislamiento, } \\
\text { las consecuencias son cambios en el } \\
\text { comportamiento laboral, emocional, } \\
\text { ambiental y otros. Por otra parte, para las } \\
\text { empresas es una ventaja porque reduce } \\
\text { gastos, contagios; se deben acoplar a un } \\
\text { conjunto de estrategias que proveen un } \\
\text { beneficio al empleado y no trabajar más } \\
\text { de lo debido. }\end{array}$ \\
\hline 5 & $\begin{array}{l}\text { (Fukushima et al., } \\
\text { 2021) }\end{array}$ & $X$ & & & $\begin{array}{l}\text { Cuantitativo, } 1239 \\
\text { trabajadores. }\end{array}$ & Cuestionario & $\begin{array}{l}\text { En la presente investigación se sugiere } \\
\text { realizar ejercicios para evitar problemas } \\
\text { ergonómicos y la importancia de seguir } \\
\text { las consultas de salud ocupacional, visto } \\
\text { a que algunas personas están } \\
\text { acostumbradas al trabajo digital, } \\
\text { evitando un régimen menos saludable. }\end{array}$ \\
\hline
\end{tabular}




\begin{tabular}{|c|c|c|c|c|c|c|}
\hline 6 & $\begin{array}{l}\text { (Zhang et al., } \\
2020)\end{array}$ & $\mathrm{X}$ & & $\begin{array}{l}\text { Cuantitativo, } 123 \text {, } \\
768 \text { trabajadores. }\end{array}$ & Cuestionario & $\begin{array}{l}\text { La investigación demostró que algunos } \\
\text { trabajadores presentan síntomas de } \\
\text { depresión, angustia y ansiedad, hasta el } \\
\text { grado de consumir bebidas alcohólicas } \\
\text { siendo algo nocivo para la salud. Es } \\
\text { necesario, instalar el seguimiento de } \\
\text { consulta psicológica en mejora de la } \\
\text { persona. }\end{array}$ \\
\hline 7 & (Nieto et al., 2020) & $\mathrm{X}$ & & $\begin{array}{l}\text { cuantitativo } \\
502 \text { españoles } \\
\text { con dolor crónico. }\end{array}$ & Cuestionario & $\begin{array}{l}\text { El proceso de investigación tuvo como } \\
\text { resultado que la mayoría de mujeres } \\
\text { sufrían de dolores intensos en diferentes } \\
\text { partes de su cuerpo debido a diferentes } \\
\text { enfermedades diagnosticada en su } \\
\text { debido tiempo, con la llegada de la } \\
\text { pandemia, esto fue deteriorado su estado } \\
\text { emocional presentando cuadros severos } \\
\text { de insomnio, ansiedad, por falta de } \\
\text { atención médica porque tenían que } \\
\text { mantener la cuarentena en sus hogares; } \\
\text { sin llegar a realizar alguna actividad } \\
\text { física. El aporte positivo es que muchas } \\
\text { personas consumieron algunos } \\
\text { medicamentes para controlar el nivel } \\
\text { alto de dolor y se incentivaros a realizar } \\
\text { estiramientos físicos en casa. Asimismo, } \\
\text { en la parte sanitaria debe prever } \\
\text { programas de rehabilitación especial } \\
\text { para los mencionados. }\end{array}$ \\
\hline 8 & $\begin{array}{l}\text { (Nelson et al., } \\
\text { 2021) }\end{array}$ & $\mathrm{X}$ & & $\begin{array}{l}\text { Cuantitativa, } 508 \\
\text { Empleados. }\end{array}$ & Cuestionario. & $\begin{array}{l}\text { Las Instituciones Educativas cumplen } \\
\text { un rol importante en tomar las medidas } \\
\text { sanitarias en prevención al contagio de } \\
\text { la COVID-19, se realizó un estudio en } \\
\text { Estados Unidos sobre posible contagio } \\
\text { en sus trabajadores, como resultado las } \\
\text { respuestas positivas en los primeros } \\
\text { meses tuvieron capacitaciones aplicando } \\
\text { medidas protocolares dentro de su } \\
\text { campo laboral; } \\
\text { como efecto no se registró algún } \\
\text { infectado. Un factor de alto interés es el } \\
\text { comportamiento y muestra de } \\
\text { responsabilidad. }\end{array}$ \\
\hline 9 & $\begin{array}{l}\text { (López-Bueno et } \\
\text { al., 2020) }\end{array}$ & $\mathrm{X}$ & & $\begin{array}{l}\text { Cuantitativa, } 516 \\
\text { padres }\end{array}$ & Cuestionario. & $\begin{array}{l}\text { La importancia de llevar una buena salud } \\
\text { en época de pandemia se ha visto con un } \\
\text { conjunto de complicaciones en las } \\
\text { personas, el resultado de la investigación } \\
\text { determina en los niños y jóvenes en } \\
\text { seguir un régimen de alimentos que } \\
\text { proveen nutrientes, minerales y } \\
\text { proteínas, s como la falta de ejercicio } \\
\text { físico, el aumento de sueño debido a la } \\
\text { pandemia. }\end{array}$ \\
\hline 10 & (Malik et al., 2021) & & $\mathrm{X}$ & $\begin{array}{l}\text { Cuantitativo, } 421 \\
\text { médicos. }\end{array}$ & Cuestionario. & $\begin{array}{l}\text { Durante la pandemia, se manifestó } \\
\text { diversos cambios de conducta en las } \\
\text { personas, se realizó una encuesta al } \\
\text { personal de salud y el resultado fue el } \\
\text { temor a permanecer en asistencia al } \\
\text { paciente con infección a causa de a } \\
\text { COVID-19; por lo tanto, el personal de } \\
\text { salud necesita un sistema de seguridad } \\
\text { con recursos protocolares para enfrentar } \\
\text { una posible deficiencia laboral en } \\
\text { atención a los enfermos. }\end{array}$ \\
\hline
\end{tabular}




\begin{tabular}{|c|c|c|c|c|c|c|}
\hline 11 & (Suka et al., 2021) & & $\mathrm{X}$ & $\begin{array}{l}\text { Cuantitativo, } \\
8000 \text { hombres y } \\
\text { mujeres. }\end{array}$ & Cuestionario. & $\begin{array}{l}\text { Después de la pandemia en Japón, se } \\
\text { invitó a una determinada cantidad de } \\
\text { personas a participar en un estudio sobre } \\
\text { su estado personal, lo cual se vio } \\
\text { minuciosamente y la respuesta fue el } \\
\text { cambio de actitud por el trabajo en casa, } \\
\text { de tal manera que se vio una } \\
\text { disminución de actividades rutinarias } \\
\text { como el ejercicio corporal; de manera } \\
\text { que deben decidir tomar acciones en } \\
\text { mejora de su salud. }\end{array}$ \\
\hline 12 & $\begin{array}{l}\text { (Linnavalli \& } \\
\text { Kalland, 2021) }\end{array}$ & $\mathrm{X}$ & & $\begin{array}{l}\text { Cuantitativo, } 82 \\
\text { familias. }\end{array}$ & cuestionarios & $\begin{array}{l}\text { En la presente trabajo se tomó una } \\
\text { encuesta a un grupo de familias, sobre } \\
\text { las consecuencias que trajo la pandemia } \\
\text { a sus vidas, como resultado se denota la } \\
\text { influencia de tensión emocional en la } \\
\text { responsabilidad, actividades laborales, } \\
\text { cuidado de los niños, como también el } \\
\text { deterioro de la relaciones de pareja. }\end{array}$ \\
\hline 13 & $\begin{array}{l}\text { (Franck et al., } \\
\text { 2021) }\end{array}$ & & $\mathrm{X}$ & $\begin{array}{l}\text { Cuantitativo, } \\
1.657 \\
\text { profesionales } \\
\text { sanitarios. }\end{array}$ & Cuestionario & $\begin{array}{l}\text { Los profesionales de salud han } \\
\text { experimentado, los cambios } \\
\text { intempestivos en la conducta, a raíz de la } \\
\text { pandemia a nivel mundial. Los síntomas } \\
\text { de tensión nerviosa, temores, agobio por } \\
\text { el exhaustivo trabajo desempeñado. Para } \\
\text { el mejoramiento en el control del estado } \\
\text { de ánimo es importante que los centros } \\
\text { de atención traten a sus médicos, } \\
\text { enfermeras y otros con un respaldo de } \\
\text { atención de perfil psicológico. }\end{array}$ \\
\hline 14 & (Yang \& Li, 2021) & & $X$ & 508 participantes. & Cuestionario. & $\begin{array}{l}\text { Las empresas deben tomar decisiones de } \\
\text { volver a producir y rescatar la économía } \\
\text { que se vio afectada en la época de } \\
\text { pandemia; a través de una encuesta en } \\
\text { China participa en el proceso conductas } \\
\text { de aportación para el retorno al } \\
\text { comercio, dando una respuesta en la } \\
\text { adaptación con un sistema protocolar y } \\
\text { evitar futuros contagios. }\end{array}$ \\
\hline 15 & $\begin{array}{l}\text { (Masatoshi et al., } \\
\text { 2021) }\end{array}$ & & $\mathrm{X}$ & $\begin{array}{l}\text { Cuantitativo, } 223 \\
\text { participantes. }\end{array}$ & Cuestionario & $\begin{array}{l}\text { La salud de algunos estudiantes } \\
\text { universitarios se ha visto afectado en la } \\
\text { salud mental, desde el principio de } \\
\text { actividades protocolares en la pandemia; } \\
\text { como resultado de la encuesta en Japón. } \\
\text { Se ha visto con un grado mayor aquellos } \\
\text { jóvenes que se encuentran viviendo } \\
\text { solos. }\end{array}$ \\
\hline 16 & $\begin{array}{l}\text { (Rogers \& } \\
\text { Cruickshank, } \\
\text { 2021) }\end{array}$ & & $X$ & $\begin{array}{l}1599 \\
\text { participantes }\end{array}$ & Cuestionario & $\begin{array}{l}\text { La participación de algunas personas en } \\
\text { la encuesta dio a conocer u alto índice de } \\
\text { sentimientos negativos, como respuesta } \\
\text { ante la cuarentena llevada a inicios de la } \\
\text { pandemia; otros factores como la salud } \\
\text { física, carencias económicas, despidos } \\
\text { intempestivos, en la parte social se } \\
\text { utiliza los medios digitales. }\end{array}$ \\
\hline 17 & $\begin{array}{l}\text { (Reinwald et al., } \\
\text { 2021) }\end{array}$ & & $\mathrm{X}$ & $\begin{array}{l}\text { Cuantitativo, } 699 \\
\text { participantes. }\end{array}$ & Cuestionario & $\begin{array}{l}\text { En la actualidad, las empresas y los } \\
\text { trabajadores se han visto afectados por el } \\
\text { tema de la COVID-19; en los resultados } \\
\text { de estudios, se ha podido ubicar lugares } \\
\text { infectados que suman una amenaza para } \\
\text { el desarrollo económico, y para las } \\
\text { personas jóvenes y de tercera edad, la } \\
\text { parte positiva es la captación de } \\
\text { empoderamiento de liderazgo de manera }\end{array}$ \\
\hline
\end{tabular}




\begin{tabular}{|c|c|c|c|c|c|c|c|}
\hline & & & & & & & $\begin{array}{l}\text { personal, evitando posibles incidentes de } \\
\text { riesgo de contagio. }\end{array}$ \\
\hline 18 & $\begin{array}{l}\text { (Sandoval-Reyes } \\
\text { et al., 2021) }\end{array}$ & X & & & $\begin{array}{l}1874 \text { participantes } \\
\text { cuantitativo }\end{array}$ & encuesta & $\begin{array}{l}\text { En países en vía de desarrollo, se ha } \\
\text { percibido que el trabajo en casa o } \\
\text { llamado trabajo remoto, no se cumple el } \\
\text { horario establecido, al contrario, una } \\
\text { persona llega a trabajar más de } 8 \text { horas; } \\
\text { en el distanciamiento social, las personas } \\
\text { desarrollaron cuadros de tensión } \\
\text { nerviosa, agotamiento, brindando la } \\
\text { producción de carga laboral. }\end{array}$ \\
\hline 19 & $\begin{array}{l}\text { (Huamán et al., } \\
\text { 2021) }\end{array}$ & & $\mathrm{X}$ & $\mathrm{X}$ & Cualitativo & & $\begin{array}{l}\text { La educación en nuestro país ha } \\
\text { evolucionado, debido a la pandemia; ha } \\
\text { realizado nuevas estrategias de } \\
\text { aprendizaje. Ejecutando el uso de } \\
\text { materiales digitales, plataformas } \\
\text { educativas y otras aplicaciones móviles, } \\
\text { respondiendo a la atención virtual } \\
\text { brindada al estudiante. El docente tiene } \\
\text { que estar capacitado y mostrar el } \\
\text { dominio del manejo de las Tics. }\end{array}$ \\
\hline 20 & $\begin{array}{l}\text { (Matacena et al., } \\
\text { 2021) }\end{array}$ & & & $X$ & $\begin{array}{l}\text { Cuantitativo, } 3630 \\
\text { residentes } \\
\text { italianos. }\end{array}$ & Encuesta. & $\begin{array}{l}\text { Como resultado de la pandemia, se ha } \\
\text { registrado cambio es de estado de ánimo } \\
\text { debido a los problemas, sociales, } \\
\text { económicos, político y otros; como } \\
\text { consecuencia, se ha detectado el } \\
\text { consumo de alimentos en exceso, a } \\
\text { cause de estrés, tensión nerviosa como } \\
\text { recuperar la tranquilidad, este tipo de } \\
\text { desequilibrio alimenticio produce } \\
\text { obesidad y puede ser perjudicial si llega } \\
\text { un control de un especialista. }\end{array}$ \\
\hline & TOTAL & 10 & 9 & 2 & & & \\
\hline & Porcentaje & $\begin{array}{l}50 \\
\%\end{array}$ & $\begin{array}{l}45 \\
\%\end{array}$ & $\begin{array}{l}10 \\
\%\end{array}$ & & & \\
\hline
\end{tabular}

Según la tabla el 100\% de los artículos analizados tienen relación a las subcategorías de la del estudio de comportamiento laboral en época de COVID-19. El $50 \%$ pertenecen a la subcategoría de comportamiento laboral (10 de 20 artículos analizados). El $45 \%$ pertenecen a la subcategoría conducta laboral (9 de 20 artículos estudiados). El 10\% corresponden a la subcategoría actitud laboral ( 2 de 20 artículos considerados para la investigación). Finalmente, el 95\% de artículos considerados para la investigación corresponden enfoque cuantitativo y el $5 \%$ al enfoque cualitativo.

En el análisis a profundidad de los 20 artículos seleccionados sobre el estudio de comportamiento laboral en el COVID-19, nos ha permitido identificar el cambió y la 
influencia del comportamiento laboral en personas de instituciones públicas y privadas, generando la siguiente discusión:

a) Se ha observado en otros estudios, algunos trabajadores que realizan sus funciones en confinamiento, han sido afectados emocionalmente ocasionando desgano de seguir llevando esta forma de rutina laboral. (Nemteanu \& Dabija, 2021). Además, se ha observado que los hijos de las personas que tienen cambios conductuales laborales también presentan problemas emocionales y de comportamiento debido a los problemas de salud mental que vienen presentando sus padres por la nueva modalidad de trabajo que vienen realizando en confinamiento.(Hosokawa \& Katsura, 2021). Luego, la modalidad del trabajo desde casa, se realizó desde años atrás, y en época de sistema se siguió este sistema para incrementar la productividad, en consecuencia, los empleados trabajaron más tiempo de lo debido, algunos jefes no toman consideración del daño que sufre el trabajador.(Magnavita et al., 2021).Por esta razón, las personas quienes desarrollan su actividad laboral desde su hogar, suelen sufrir problemas físicos, por el reposo sin tener actividad de ejercicio corporal.(Fukushima et al., 2021).También, el consumo de bebidas alcohólicas se elevó, en algunas personas quienes sufrían de un nivel de depresión y ansiedad.(Zhang et al., 2020). Por esta razón, algunos pacientes durante el confinamiento, experimentaron el dolor intenso en su cuerpo, por diagnósticos médicos antes de la pandemia, lo único que hicieron fue consumir medicamentos para calmar su malestar y realizando ejercicios desde su hogar(Nieto et al., 2020). Por una parte, es necesario aplicar las medidas de prevención de contagio de la COVID-19, de esta manera disminuirán el riesgo en sus trabajadores. (Nelson et al., 2021). Es conveniente, seguir una buena calidad de vida en el consumo de alimentos que no generan enfermedades como obesidad y otros. López. Luego, algunos padres de familia han demostrado tener problemas de relaciones afectivas con su pareja, debido a la tensión nerviosa a causa de la pandemia, el comportamiento en sus hijos ha cambiado(Linnavalli \& Kalland, 2021). Es conveniente, limitar las jornadas laborales remotas, de esta manera reducirán el cansancio y tener tiempo en su vida cotidiana. (Sandoval-Reyes et al., 2021).

b) Actualmente, los problemas del estado emocional en las personas, se manifiestan con incremento de ansiedad, depresión y el control de emociones, iniciando un problema psiquiátrico.(Awano et al., 2020). Igualmente, en el personal de salud tuvo variaciones de conducta referente al temor en atención a pacientes de COVID-19; la implementación de 
desarrollar programas protocolares en beneficio de los mencionados.(Malik et al., 2021). Además, se ha visto la falta de actividades deportivas físicas en personas con trabajo en casa, limitando a tener buena condición de salud.(Suka et al., 2021).Al mismo tiempo, se ha incrementado otros síntomas como; temores, fatiga, por trabajos fuera de hora, el control psicológico en los trabajadores es muy importante para el tratamiento de las personas. (Franck et al., 2021).Es conveniente, adaptar a los trabajadores con sistemas protocolares, sensibilizar en la conducta de responsabilidad. (Yang \& Li, 2021). Con referencia, a estudios en alumnos de la universidad, se ha encontrado un mayor índice de problemas en la salud mental; a consecuencia de guardar cuarentena y no asistir a su centro de actividades académicas.(Masatoshi et al., 2021).También, estos problemas en la salud, presentan la negatividad de desarrollar con naturalidad las actividades de trabajo debido a los cambios sociales, económicos y laboral.(Rogers \& Cruickshank, 2021).En síntesis, el líder debe ser muy comunicativo con las personas que laboran para una empresa, evitando la infección, de esta manera habrá un resguardo en el desarrollo de la producción y la parte económica(Reinwald et al., 2021).

c) Por lo tanto, el cambio de la educación, se lleva en casa por medio de aplicaciones digitales, las cuales los docentes deben estar capacitados para responder la necesidad de aprendizaje del estudiante(Huamán et al., 2021).

d) Por último, se registra los problemas de estado de ánimo, el control de no equilibrar la alimentación(Matacena et al., 2021).

\section{CONCLUSIÓN O CONSIDERACIONES FINALES}

El comportamiento laboral durante la pandemia de la COVID-19 transformó la vida de muchas personas, al observar las muertes de manera trágica. A nivel mundial; la salud mental de la población se percibió síntomas de estrés, desequilibrio alimenticio, cansancio, ergonomía, temor a ser contagiado por este nuevo virus, problemas de relaciones matrimoniales y familiares debido al confinamiento. Se ha individualizado el desempeño laboral a consecuencia de la dificultad de comunicación, obteniendo retrasos de entrega en el cumplimiento de la labor encomendada. El teletrabajo fue una manera de sobrellevar la producción con la responsabilidad planificando el sostenimiento del capital de inversión en las empresas. Asimismo, inician nuevas estrategias de motivación a los empleados en dar el tiempo necesario para cumplir con las metas de ganancia. 
El plan de protocolo es necesario para prevenir la infección de la covid-19 enfrentando la informalidad laboral en algunos sectores, donde las personas se arriesgan a trabajar estando en el proceso de recuperación debido a la tensión económica; por lo tanto, la población debe ser responsable en asumir el cumplimiento del resguardo sanitario ante un nuevo rebrote, a través de la comunicación efectiva con el personal de salud son bien recibidas por los gobiernos, quienes decretan nuevas estrategias de obedecer las normas de salubridad, a lo contrario se verían sancionados.

Ante la emergencia mundial; la salud en el ámbito social, económico y político se vio afectada, por la falta de preparación del personal y debilidad del sistema de salud en atención a pacientes infectados con la covid-19, en el aspecto social se observó la desigualdad y los despidos intempestivos a personas de bajos recursos económicos; la crisis monetaria mundial se devaluó debido a que algunos países no tenían un presupuesto implementando ante esta emergencia; dentro de la política gubernamental se luchaba por realizar modificaciones en atender y evitar el desastre de extinción de su población.

El nuevo reto de recuperar la estabilidad económica a nivel social se estará rescatando lentamente ante esta emergencia sanitaria, la dependencia cibernética ha influido en el comportamiento laboral, es necesario ajustar el proceso de reactivación de actividades laborales cumpliendo normas legales de acuerdo al país o gobierno que lo establezca, manteniendo el soporte de un medio ambiente sostenible en contra de la contaminación, el proceso de la educación y el sistema de salud tiene que mejorar en una nueva oportunidad de alinear para el servicio a favor del ciudadano.

\section{LISTA DE REFERENCIAS}

Antezana Llaveta, G., \& Arandia-Guzmán, J. (2020). SARS-CoV-2: estructura, replicación y mecanismos fisiopatológicos relacionados con COVID-19. Gaceta Medica Boliviana, 43(2), 172-178. https://doi.org/10.47993/gmb.v43i2.85

Awano, N., Oyama, N., Akiyama, K., Inomata, M., Kuse, N., Tone, M., Takada, K., Muto, Y., Fujimoto, K., Akagi, Y., Mawatari, M., Ueda, A., Kawakami, J., Komatsu, J., \& Izumo, T. (2020). Anxiety, depression, and resilience of healthcare workers in japan during the coronavirus disease 2019 outbreak. Internal Medicine, 59(21), 2693-2699. https://doi.org/10.2169/internalmedicine.5694-20

Bogliacino, F., Codagnone, C., Montealegre, F., Folkvord, F., Gómez, C., Charris, R., Liva, G., Lupiáñez-Villanueva, F., \& Veltri, G. A. (2021). Negative shocks predict 
change in cognitive function and preferences: assessing the negative affect and stress hypothesis. Scientific Reports, 11(1), 1-10. https://doi.org/10.1038/s41598021-83089-0

Bradley, J., Ruggieri, A., \& Spencer, A. H. (2021). Twin Peaks: Covid-19 and the labor market. European Economic Review, 138(July), 103828. https://doi.org/10.1016/j.euroecorev.2021.103828

Bytyçi, S., Shala, V., Ziberi, B., \& Myftaraj, E. (2021). Transforming traditional business into online: The impact of COVID-19 pandemic on consumer behavior. Journal of Governance and Regulation, 10(2 Special issue), 300-308. https://doi.org/10.22495/JGRV10I2SIART10

Converso, D., Bruno, A., Capone, V., Colombo, L., Falco, A., Galanti, T., Girardi, D., Guidetti, G., Viotti, S., \& Loera, B. (2021). Working during a pandemic between the risk of being infected and/or the risks related to social distancing: First validation of the saph@w questionnaire. International Journal of Environmental Research and Public Health, 18(11). https://doi.org/10.3390/ijerph18115986

Franck, E., Haegdorens, F., Goossens, E., van Gils, Y., Portzky, M., Somville, F., Abuawad, M., Slootmans, S., \& Van, P. (2021). The Role of Coping Behavior in Healthcare Workers' Distress and Somatization During the COVID-19 Pandemic. Frontiers in Psychology, 12, 1-12. https://doi.org/10.3389/fpsyg.2021.684618

Fukushima, N., Machida, M., Kikuchi, H., Amagasa, S., Hayashi, T., Odagiri, Y., Takamiya, T., \& Inoue, S. (2021). Associations of working from home with occupational physical activity and sedentary behavior under the COVID-19 pandemic. Journal of Occupational Health, 63(1), e12212. https://doi.org/10.1002/1348-9585.12212

Hosokawa, R., \& Katsura, T. (2021). Maternal work-life balance and children's social adjustment: The mediating role of perceived stress and parenting practices. International Journal of Environmental Research and Public Health, 18(13). https://doi.org/10.3390/ijerph18136924

Huamán, L., Torres, L. A., Amancio, A. M., \& Sánchez, S. (2021). apuntesuniversitarios.upeu.edu.pe. 11(3), 45-60.

Krasna, H., Czabanowska, K., Beck, A., Cushman, L. F., \& Leider, J. P. (2021). Labour market competition for public health graduates in the United States: A comparison 
of workforce taxonomies with job postings before and during the COVID-19 pandemic. International Journal of Health Planning and Management, 36(December 2020), 151-167. https://doi.org/10.1002/hpm.3128

Krisjane, Z., Apsite-Berina, E., Berzins, M., Skadins, T., \& Burgmanis. (2020). WorkLife Balance During the Covid-19 Outbreak: the Case of Latvia. Baltic Region, 12(4), 40-60. https://doi.org/10.5922/2079-8555-2020-4-3

Linnavalli, T., \& Kalland, M. (2021). Impact of COVID-19 Restrictions on the SocialEmotional Wellbeing of Preschool Children and Their Families. Education Sciences, 11(8), 1-12. https://doi.org/10.3390/educsci11080435

López-Bueno, R., López-Sánchez, G. F., Casajús, J. A., Calatayud, J., Gil-Salmerón, A., Grabovac, I., Tully, M. A., \& Smith, L. (2020). Health-Related Behaviors Among School-Aged Children and Adolescents During the Spanish Covid-19 Confinement. Frontiers in Pediatrics, 8, 1-11. https://doi.org/10.3389/fped.2020.00573

Magnavita, N., Tripepi, G., \& Chiorri, C. (2021). Telecommuting, off-time work, and intrusive leadership in workers' well-being. International Journal of Environmental Research and Public Health, 18(7), 1-10. https://doi.org/10.3390/ijerph18073330

Malik, S., Ullah, I., Muhammad, I., Kwasi, D., Chung-Ying, L., Pakpour, A. H., Griffiths, M. D., Ur, I., \& Minhas, R. (2021). Fear of COVID-19 and workplace phobia among Pakistani doctors: A survey study. BMC Public Health, 21(1), 1-9. https://doi.org/10.1186/s12889-021-10873-y

Mangiavacchi, L., Piccoli, L., \& Pieroni, L. (2021). Fathers matter: Intrahousehold responsibilities and children's wellbeing during the COVID-19 lockdown in Italy. Economics Human Biology, 42, 101016. https://doi.org/10.1016/j.ehb.2021.101016

Masatoshi, T., Mashizume, Y., \& Takahashi, K. (2021). Mental health crisis and stress coping among healthcare college students momentarily displaced from their campus community because of covid-19 restrictions in japan. International Journal of Environmental Research and Public Health, 18(14), 1-12. https://doi.org/10.3390/ijerph18147245

Matacena, R., Zenga, M., D’addario, M., Mari, S., \& Labra, M. (2021). COVID-19 as an 
opportunity for a healthy-sustainable food transition. An analysis of dietary transformations during the first Italian lockdown. Sustainability (Switzerland), 13(10), 1-22. https://doi.org/10.3390/su13105661

Moher, D., Liberati, A., Tetzlaff, J., \& Altman, D. G. (2009). Preferred reporting items for systematic reviews and meta-analyses: the PRISMA statement. Journal of Clinical Epidemiology, 62(10), 1006-1012. https://doi.org/10.1016/j.jclinepi.2009.06.005

Nelson, T. L., Fosdick, B. K., Biela, L. M., Schoenberg, H., Mast, S., McGinnis, E., Young, M. C., Lynn, L., Fahrner, S., Nolt, L., Dihle, T., Quicke, K., Gallichotte, E., Fitzmeyer, E., Ebel, G. D., Pabilonia, K., Ehrhart, N., \& Vandewoude, S. (2021). Association between COVID-19 Exposure and Self-reported Compliance with Public Health Guidelines among Essential Employees at an Institution of Higher Education in the US. JAMA Network Open, 4(7), 1-12. https://doi.org/10.1001/jamanetworkopen.2021.16543

Nemteanu, M. S., \& Dabija, D. C. (2021). The influence of internal marketing and job satisfaction on task performance and counterproductive work behavior in an emerging marketing during the covid-19 pandemic. International Journal of Environmental Research and Public Health, 18(7). https://doi.org/10.3390/ijerph18073670

Nieto, R., Pardo, R., Sora, B., Feliu-Soler, A., \& Luciano, J. V. (2020). Impact of COVID19 Lockdown Measures on Spanish People with Chronic Pain: An Online Study Survey. Journal of Clinical Medicine, 9(11), 1-15. https://doi.org/10.3390/jcm9113558

Qin, W., Li, L., Zhu, D., Ju, C., Bi, P., \& Li, S. (2021). Prevalence and risk factors of depression symptoms among Chinese seafarers during the COVID-19 pandemic: A cross-sectional study. BMJ Open, 11(6), 1-8. https://doi.org/10.1136/bmjopen2021-048660

Reinwald, M., Zimmermann, S., \& Kunze, F. (2021). Working in the Eye of the Pandemic: Local COVID-19 Infections and Daily Employee Engagement. Frontiers in Psychology, 12, 1-10. https://doi.org/10.3389/fpsyg.2021.654126

Reuter, M., Dragano, N., \& Wahrendorf, M. (2021). Working while sick in context of regional unemployment: A Europe-wide cross-sectional study. Journal of 
Epidemiology and Community Health, 75(6), 574-580. https://doi.org/10.1136/jech-2020-214888

Rogers, S. L., \& Cruickshank, T. (2021). Change in mental health, physical health, and social relationships during highly restrictive lockdown in the COVID-19 pandemic: Evidence from Australia. PeerJ, 9, 1-28. https://doi.org/10.7717/peerj.11767

Sandoval-Reyes, J., Idrovo-Carlier, S., \& Duque-Oliva, E. J. (2021). Remote work, work stress, and work-life during pandemic times: A Latin America situation. International Journal of Environmental Research and Public Health, 18(13), 112. https://doi.org/10.3390/ijerph18137069

Suka, M., Yamauchi, T., \& Yanagisawa, H. (2021). Changes in health status, workload, and lifestyle after starting the COVID-19 pandemic: a web-based survey of Japanese men and women. Environmental Health and Preventive Medicine, 26(1), 1-11. https://doi.org/10.1186/s12199-021-00957-x

Tsurugano, S., Nishikitani, M., Inoue, M., \& Yano, E. (2021). Impact of the COVID-19 pandemic on working students: Results from the Labour Force Survey and the student lifestyle survey. Journal of Occupational Health, 63(1), e12209. https://doi.org/10.1002/1348-9585.12209

Yang, X., \& Li, J. (2021). Analysis of Decision Making of Energy Enterprises on Adaptive Behavior Amid COVID-19. Frontiers in Energy Research, 9(July), 110. https://doi.org/10.3389/fenrg.2021.716171

Yiwen, F., \& Hahn, J. (2021). Job insecurity in the covid-19 pandemic on counterproductive work behavior of millennials: A time-lagged mediated and moderated model. International Journal of Environmental Research and Public Health, 18(16). https://doi.org/10.3390/ijerph18168354

Zhang, X.-R., Huang, Q.-M., Wang, X.-M., Cheng, X., Li, Z.-H., Wang, Z.-H., Zhong, W.-F., Liu, D., Shen, D., Chen, P.-L., Song, W.-Q., Wu, X.-B., Yang, X., \& Mao, C. (2020). Prevalence of anxiety and depression symptoms, and association with epidemic-related factors during the epidemic period of COVID-19 among 123,768 workers in China: A large cross-sectional study. Journal of Affective Disorders, 277, 495-502. https://doi.org/10.1016/j.jad.2020.08.041 